\title{
A Study on Predicting the Length of Hospital Stay for Chinese Patients With Ischemic Stroke Based on the XGBoost Algorithm
}

\section{Chen Rui}

Hebei University of Technology

Shengfa Zhang ( $\square$ zhangshengfa1988@hotmail.com )

Beijing Normal University https://orcid.org/0000-0001-7980-1326

Jie Li

Hebei University of Technology

Dongwei Guo

Hebei University of Technology

Weijun Zhang

Hebei University of Technology

\section{Donghua Tian}

Hebei University of Technology

Linni Gu

Beijing Normal University

Zhiyong Qu

Beijing Normal University

Xiaohua Wang

Beijing Normal University

\section{Research article}

Keywords: Ischemic stroke, XGBoost algorithm, Length of hospital stay (LOS), Machine Learning (ML) Model

Posted Date: March 23rd, 2021

DOI: https://doi.org/10.21203/rs.3.rs-344337/v1

License: (c) (i) This work is licensed under a Creative Commons Attribution 4.0 International License.

Read Full License 
A study on predicting the length of hospital stay for Chinese patients with ischemic stroke based on the XGBoost algorithm

Chen Rui ${ }^{1}$, Shengfa Zhang ${ }^{2}$, Jie $\mathrm{Li}^{1}$, Dongwei Guo ${ }^{1}$, Weijing Zhang ${ }^{3}$, Donghua Tian $^{3}$, Linni $\mathrm{Gu}^{3}$, Zhiyong $\mathrm{Qu}^{3}$, Xiaohua Wang ${ }^{3}$

Correspondence: zhangshengfa1988@hotmail.com 


\section{Abstract \\ Background}

China faced the most significant challenge from stroke because it imposes a heavy burden on families, national health services, social services, and the economy. The length of hospital stay (LOS) was an essential indicator of utilization of medical services and was usually used to assess the efficiency of hospital management and patient quality of care. This study established a prediction model based on the machine learning algorithm to predict the ischemic stroke patients' LOS.

\section{Methods}

A total of 18,195 ischemic stroke patients' electronic medical records and 28 attributes were extracted from electronic medical records in a large comprehensive hospital in China. After preprocessing the data and feature selection, the XGBoost algorithm was used for building a machine learning model. The 10-fold cross-validation was used for model validation. The accuracy (ACC), recall rate (RE) and F1 measure were used to evaluate the performance of the prediction model of LOS of ischemic stroke patients. Finally, the XGBoost algorithm was used to identify and remove irrelevant features by ranking all attributes based on feature importance.

\section{Results}

The average ACC, RE and F1 measure were 0.96, 0.82 and 0.79, respectively, under the 10-fold cross-validation. According to the analysis of the importance of features, the LOS of ischemic stroke patients was affected by demographic characteristics, past medical history, admission examination features, and operation characteristics. Finally, the features, including NIHSS, MRS, Hemiplegia aphasia, age, BMI and TIA etc. were found to be the top ten features in importance in predicting the LOS of ischemic stroke patients.

\section{Conclusions}

The XGBoost algorithm was an appropriate machine learning method for predicting the LOS of patients with ischemic stroke. Based on the prediction model, an intelligent medical management prediction system could be developed to predict the LOS based on ischemic stroke patients' electronic medical records.

Key words Ischemic stroke; XGBoost algorithm; Length of hospital stay (LOS); Machine Learning (ML) Model 


\section{Background}

Stroke, as the second most common cause of death worldwide and the leading cause of acquired disability in adults, was a kind of acute cerebrovascular disease characterized by the focal neurological deficit, mainly including ischemic stroke and hemorrhagic stroke[1,2]. Stoke had become the second most fatal disease in the world and its disability-adjusted life years (DALYs) and the years of life lost (YLLs) due to stroke ranked third globally [3, 4]. China faced the biggest stroke challenge from stroke in the world. In China, cerebrovascular disease's mortality rate was 149.49 per 100000 , accounting for 1.57 million deaths in 2018[5]. The 2016 global burden of disease (GBD) study estimated that the estimated lifetime risk of stroke in China from 25 was the highest, as high as $39.3 \%$ [4].

Ischemic stroke, which is the most common stroke type and accounts for $70 \%-80 \%$ of strokes, was caused by cerebral artery occlusion [6]. In China, the incidence rate of ischemic stroke has been rising, especially for young people under 45 years old, which has brought a heavy burden to the family, national health, social services, and economy $[7,8]$. The increasing trend of ischemic stroke incidence rate was young patients, rather than the elderly, which had caused a heavier social and economic burden. It had previously been reported that among the Chinese aged 15-45, the incidence of stroke were between $18-142$ per 100,000 in male and between $23-95$ per 100,000 in female, and between $9.77-13.44 \%$ for all stroke patients in China were under 45 years of age [9]. Although the management of ischemic stroke had improved over time, it came with a high economic burden. In 2013, the results of the particular investigation of cerebrovascular disease epidemiology showed that the weighted rate of ischemic stroke was $854.5 / 10$ million, the weighted rate was $166.9 / 10$ million, and the death weighted rate was $56.5 / 10$ million, regardless of the prevalence rate $(155 \%)$ or the incidence rate (31.6\%) all increased significantly[10]. According to the data, in 2017, the average hospitalization expenses of ischemic stroke patients in China were 18525 yuan, an increase of $118 \%$ compared with 2007 and the average annual growth rate of ischemic stroke far exceeded the growth rate of GDP[11]. More than half of the costs associated with ischemic stroke were related to hospitalization. Notably, one of the best predictive markers for hospitalization expenses among stroke patients was length of hospital stay (LOS). Therefore, it was reasonable to focus on sustainable quality improvement efforts to reduce LOS. Accurate prediction of LOS had become increasingly important for health care systems, and reducing the LOS had the potential for considerable savings in the public hospital system. Previous studies showed that LOS was associated with stroke severity, complications during the hospital stay, stroke subtype, course of diseases, treatment, hypertension, diabetes mellitus, and smoking, age etc. At present, studies mainly focused on the analysis of influencing factors of hospitalization costs for ischemic stroke, and further studies on the prediction of LOS were relatively few [12, 13]. The existing studies were mainly based on traditional statistical analysis methods, and the research starting from data mining methods was lagging behind[14]. The existing studies mainly based on traditional statistical analysis methods, and the research starting from data mining methods was relatively lagging behind. However, 
with intelligent medical technology development, data mining has been widely used in the medical field due to its advantages of efficiently extracting value information from massive medical data. Therefore, this study aimed to establish a prediction model based on the machine learning algorithm to predict ischemic stroke patients' LOS.

The benefits of measuring hospital service level were economical and extended to other medical care aspects, including patients themselves. First, predicting LOS enabled hospitals to identify patients with a high risk of long-term hospitalization and then use the optimized treatment plan to treat them or carry out early intervention to prevent other complications. Second, predicting LOS could lead to earlier discharge to the home, or a cheaper medical institution supported by community nurses and doctors. Specifically, as an important index to measure inpatients' resource consumption, accurately predicting patients' service level provides a better management means for the hospital to improve the utilization rate of resources. Therefore, predicting patients' LOS could help medical service institutions enhance patient satisfaction, make more effective use of human resources and facilities, reduce treatment costs and improve sustainability.

In recent years, a major contributor to realizing these improvements in health care outcomes had been the increasing use of big data analytics. Previous studies employed data analytic approaches, such as regression methods, naive Bayes, multi-layer neural network (MLP), K-Nearest Neighbors (KNN) algorithm, classification and regression tree (CART) and support vector regression, to develop a model for the early prediction of patients' LOS [15-17]. The objective of this study was to describe the development and validation of a new machine learning model to predict the LOS of patients at the time of admission.

The XGBoost (Extreme Gradient Boosting) algorithm, a machine learning algorithm, could improve the integration of multiple decision trees by using the gradient promotion method, which has the characteristics of high accuracy, difficulty in overfitting and scalability. The XGBoost also could deal with high-dimensional sparse features in a distributed manner. At present, the XGBoost algorithm had been widely used in fields such as short-term passenger demand, fault monitoring and commercial default prediction [18-20]. In recent years, the XGBoost algorithm had been gradually applied in the field of health care. and had been involved in the prediction of fraud risk, heart disease, pneumonia, diabetes and cardiovascular disease and other aspects [21, 22]. Therefore, it was feasible and practical to apply the XGBoost algorithm to predict of hospitalization days of ischemic stroke patients.

This study aims to establish an integrated prediction model based on the XGBoost algorithm to predict the LOS of ischemic stroke patients so as to provide better decision support for hospital management of medical resources and doctors' choice of the treatment plan, thus saving the treatment costs of patients in the hospital and improving the later rehabilitation effect.

\section{Methods}

Several steps were used in this study, as showed in Figure.1.

(1) The medical record attributes of ischemic stroke patients were extracted from electronic medical records. 
(2) Several methods were used for data pre-processing.

(3) Feature selection was used for selecting the key subset of features to reduce the dimensionality of the training problem.

(4) The XGBoost algorithm was used for building a machine learning (ML) Model.

(5) The 10-fold cross-validation method was used for model validation.

(6) Selected features for building the model for predicting LOS.

\subsection{The dataset}

The dataset of this study included adult patients who were admitted between 2017 and 2018 in the Cangzhou Central Hospital in Hebei Province, China. A total of 18,195 cardiac patients' records and 28 attributes were extracted from electronic medical records. These attributes were collected prospectively by experienced physicians and trained nurses. The attributes of the dataset included demographic characteristics, past medical history, admission examination characteristics and operation characteristics. Admission diagnosis and the primary treating physician were reviewed to verify selection criteria. This study included all patients who were admitted to the cardiac wards under the clinical cardiology service. Patients who were admitted under other services in the cardiac wards for non-cardiac-related admitting diagnosis were excluded.

The attributes were selected in the final model based on their information gain. The LOS was determined by subtracting the discharge date from the admission date. According to the medical research, the length of hospitalization for ischemic stroke in the range of 7-14 days was a protective factor for the adverse outcome after discharge. Meanwhile, in combination with the provisions of China's medical insurance policy, the length of hospitalization for ischemic stroke in the range of 1-7 days, 8-14 days, and more than 14 days were discretized for prediction, and the data ratio of the three types was 1.7:3.9:1.

By eliminating the invalid variables, missing value filling and data integration, 28 characteristic variables and label variables of length of stay were extracted, and 18,195 training samples of ischemic stroke patients were collected, forming a $28 \times 18195$ feature-sample training data set matrix. The 28 characteristic variables could be divided into demographic characteristics, past medical history, admission examination information and operation features, and the specific features were shown in Table 1.

The LOS for each inpatient admission was calculated by subtracting the date and time of admission from the date of discharge. The time elapsed between the previous inpatient admission and the current index admission was calculated by the date difference between them.

\subsection{Data pre-processing}

Since the data of ischemic stroke patients were manually entered, there might be missing values, abnormal values, repeated values, etc. These abnormal data affected the real information in the model learning data and had adverse effects on the final performance of the model. Therefore, the data should be preprocessed before model 
training. A few data pre-processing steps were taken before analyzing the data:

(1) All records of the same patient were merged if the patient had multiple hospitalizations on the same day to the same medical unit. That applied to both medical and surgical inpatients.

(2) Admissions that were recorded as having occurred within 24 hours of the immediately prior index discharge were excluded from the admission set.

(3) Missing value referred to the missing data caused by data loss in the process of data entry or extraction. There were two ways to deal with the missing value. For the features with more missing values, the deletion operation was usually used, and the features with less missing values could be filled by means of means, modes, median and other methods. In this study, the missing values in the data of patients with ischemic stroke were less, so the mean value was used by the row fill method.

(4) Abnormal value referred to that the data in a feature was far beyond the feature' overall distribution. In practice, it might be caused by an error in the input, and the abnormal value might have a significant impact on the prediction results. When the dimensions and orders of magnitude of different features were different, using the original data would make the algorithm biased to additional features, affecting the prediction performance of the model. Therefore, this study used standard normalization to standardize the data.

(5) Inconsistent and/or erroneous components, such as age discrepancies, or a discharge date that preceded the admission date, were excluded.

(6) The dataset was separated randomly into a training set of 14556 admissions (80\%), and a test (holdout/validation) set of 3639 admissions (20\%).

\subsection{Build ML Model using the XGBoost Algorithm}

The ML model was developed and validated using the data set consisting of patients with complete data ( $98.2 \%$ of the full dataset) to compare the modeling procedures with an identical patient population. We used an 80/20 random split for the training and testing datasets.

\subsubsection{The XGBoost algorithm}

In this study, the XGBoost (Extreme Gradient Boosting) algorithm was used to build the model that achieves the best performance for our purpose.

The XGBoost algorithm, which was improved by T.Q.Hen in 2015 basing on the Gradient Boosting Decision Tree, was based on CART classification and regression tree, whose main idea was to generate a base learning device based on the selection of partial sample features, segment fitting residual in the process, and finally form a comprehensive model for prediction.

A single CART tree could train the corresponding prediction score of $\mathrm{F}(\mathrm{X})$ for each sample. The XGBoost algorithm was an additive model obtained by integrating the prediction results of multiple carts, as shown in Equation (1).

$$
\widehat{y_{l}}=\phi\left(X_{i}\right)=\sum_{k=1}^{K} f_{k}\left(X_{i}\right), f_{k} \in F
$$


$\mathrm{X}_{\mathrm{i}}$ represents the ith hospitalized patients with ischemic stroke. $\widehat{y}_{l}$ represents the predicted results of $X_{i}$. K represents all cart tree based learners and F was the space of the CART tree. According to Equation (1), the XGBoost algorithm maps it to the corresponding leaf node by applying K CART decision rules, and adds up the mapping scores of each leaf node to obtain the final classification prediction score of the sample. The XGBoost algorithm of the objective function includes two parts. The first part of predicted value $\widehat{y}_{l}$ with real value $y_{i}$ between training errors, measured by its model was in accordance with the law of training data. The second part was the penalty term of model complexity, namely regularization function, so as to avoid model overfitting and make the model more robust. The objective function was shown in Equation (2).

$$
\operatorname{Obj}(\Theta)=\mathrm{L}(\Theta)+\Omega(\Theta)=\sum_{i=1}^{n} l\left(y_{i}, \widehat{y}_{l}\right)+\sum_{k=1}^{K} \Omega\left(f_{k}\right)
$$

This algorithm used the iterative method to solve the model, starting from the constant prediction, and each iteration added a new function to the model. $\widehat{y}_{l}^{(t)}$ represents the ith sample's prediction score in the t-th iteration, as shown in Equation (3).

$$
\widehat{y}_{l}^{(t)}=\sum_{k=1}^{t} f_{k}\left(x_{i}\right)=\widehat{y}^{(t-1)}+f_{t}\left(x_{i}\right)
$$

In each iteration, $f_{t}\left(x_{i}\right)$ was obtained by optimizing the objective function, as shown in the Equation (4).

$$
\mathrm{Obj}^{(t)}+\sum_{i=1}^{t} \Omega\left(f_{i}\right)=\sum_{i=1}^{n} l\left(y_{i}, \widehat{y}_{l}^{(t-1)}+f_{t}\left(x_{i}\right)\right)+\Omega\left(f_{t}\right)
$$

The XGBoost algorithm used Taylor's second-order expansion to optimize the objective function. If the first derivative $g_{i}=\partial_{\widehat{y}_{l}}(t-1) l\left(y_{i}, \widehat{y}_{l}^{(t-1)}\right)$ and the second derivative $h_{i}=\partial_{\widehat{y}_{l}}^{2}{ }^{(t-1)} l\left(y_{i}, \widehat{y}_{l}^{(t-1)}\right)$ were defined, the approximate objective function can be obtained, as shown in the Equation (5)

$$
\widetilde{\mathrm{Ob}}^{(t)} \cong \sum_{i=1}^{n}\left[g_{i} f_{t}\left(x_{i}\right)+\frac{1}{2} h_{i} f_{t}^{2}\left(x_{i}\right)\right]+\Omega\left(f_{t}\right)
$$

In the objective function, $\Omega\left(f_{t}\right)=\gamma \mathrm{T}+\frac{1}{2} \lambda \sum_{j=1}^{T} \omega_{j}{ }^{2}$ was a regularization term, which defines the complexity penalty term according to the number $\mathrm{T}$ of leaf nodes and the weight of the leaf nodes. Here, $\gamma$ and $\lambda$ were the penalty coefficients of them to control the degree of regularization.

Finally, define the sample set in each leaf as $I_{j}=\left\{i \mid q\left(X_{i}\right)=j\right\}$, then the objective function can be rewritten according to the leaf node, as shown in Equation (6). 


$$
\mathrm{Obj}^{(t)}=\sum_{j=1}^{T}\left[\left(\sum_{i \in I_{j}} g_{i}\right) \omega_{j}+\frac{1}{2}\left(\sum_{i \in I_{j}} h_{i}+\lambda\right) \omega_{j}{ }^{2}\right]+\gamma \mathrm{T}
$$

If $G_{j}$ is defined as $G_{j}=\sum_{i \in I_{j}} g_{i}$ and $H_{j}=\sum_{i \in I_{j}} h_{i}$, there was:

$$
\mathrm{Obj}^{(t)}=\sum_{j=1}^{T}\left[G_{j}+\frac{1}{2}\left(H_{j}+\lambda\right) \omega_{j}^{2}\right]+\gamma \mathrm{T}
$$

If the structure of the $\mathrm{t}$-th tree was given as $q(X)$, the optimal weight of leaves could be obtained from the partial derivative formula $\partial_{\omega_{j}} \mathrm{Obj}^{(t)}=0$ as $\omega_{j}{ }^{*}=-\frac{G_{j}}{H_{j}+\lambda}$, and then the optimal objective function was shown in Equation (8).

$$
\mathrm{Obj}^{(t)}=-\frac{1}{2} \sum_{j=1}^{T} \frac{G_{j}^{2}}{H_{j}+\lambda}+\gamma \mathrm{T}
$$

\subsubsection{Training and Test}

For the training process, to ensure the same splits for all algorithms, we partitioned the (randomly reordered) training data set ( $80 \%$ of the whole sample) into 10 parts with an equal number of observations in each part. We indexed the observations in each part, so the index runs from 1 to 10; after that we took the part 1 to test the model and the other 9 parts to train the model (part 2 to part 10). This procedure was repeated taking part 2 to test the model and the other 9 parts to train the model, and so on, until we reached part 10 . Hence, we obtained the trained model from a 10 -fold cross-validation. After that, we used the hold-out testing data set ( $20 \%$ of the whole sample) to calculate the AUC and its standard error with the analytical formula of Hanley and McNeil (1982).

\subsubsection{Model building}

The 10-fold cross-validation was used to train the model of hospitalization days of cerebral infarction. The main steps of model building were as follows: (1) The data set $\mathrm{D} x_{i}$ of patients with cerebral infarction was divided into 10 mutually exclusive subsets with similar size, and the data distribution in each subgroup was as consistent as possible, thus 10 sample subsets $\mathrm{D} x_{i j}(\mathrm{~J}=1,2, \ldots)$ were obtained; (2) for the $j$-th training subset $D x_{i j}$, it was used as the test set, and the union of the other nine sample subsets was used as the training set, and the XGBoost algorithm was used to train the model; (3) repeat the second step, train 10 sample subsets in turn, and got five base prediction models $M i(I=1,2, \ldots)$; (4) using bagging integration idea, the average value of the performance measures of the five prediction models was used as the performance index of the prediction model of Ischemic stroke hospitalization days; (5) the grid search of sklearn Gridsearchcv in Python was used to determine the parameters in the model, and finally the prediction model of Ischemic stroke hospitalization days was obtained. The results were shown in Table. 2

\subsection{Model evaluation and validation}


In this paper, combined with medical research and China's medical insurance policy, it is a typical three classification problem to convert the length of stay of ischemic stroke into discrete variables of three stages. The definition of prediction results $(i=1,2,3 ; j=$ $1,2,3)$ represents the number of samples predicted from class $i$ to class $j$, where $I$ represent the real value category of the sample, and $j$ represents the category of the predicted value of the sample. In this paper, the accuracy (ACC), recall rate (RE) and $F_{1}$ measure were used to evaluate the performance of the prediction model of LOS of ischemic stroke patients.

$$
\begin{gathered}
A C C=\sum_{i=j=1}^{3} P_{i j} / \sum_{i, j=1}^{3} P_{i j} \\
R E=\frac{1}{3} \sum_{i=j=1}^{3} P_{i j} / \sum_{j=1}^{3} P_{i j} \\
F_{1}=2 * R E * P R E /(R E+P R E)
\end{gathered}
$$

\subsection{Relevant features ranking}

The information gain method was used to determine the most important features, which can significantly affect the accuracy of the model. The technique could sort all attributes according to their importance and identify and remove irrelevant features.

\section{Results}

\subsection{The baseline characteristics of patients with ischemic stroke among different LOS group.}

A total of $(18,195)$ cardiac visits for patients with ischemic stroke who were admitted to the cardiac center from 2017 and 2018 were analyzed. Of these, the baseline features were shown in Table 3.

\subsection{The performance of the different machine learning models evaluated}

In this study, the data sets of patients with ischemic stroke were predicted 10 times on the prediction model to verify the stability of the model. The three evaluation indexes of the prediction results were shown in Table 4.

Among them, the ACC was 0.96, which indicates that the model had accurate prediction performance for LOS in each stage. The RE reached 0.82, which indicates that the model could better identify the hospitalization time in each stage in the data. The $F_{1}$ measure was 0.79 , indicating that the model still had good performance when the comprehensive recall rate and accuracy rate were used. The standard deviation of ACC, $\mathrm{RE}$ and $\mathrm{F}_{1}$ measure were all less than 0.05 , which indicates that the model has good stability. In conclusion, the integrated model based on the XGBoost algorithm could effectively predict the hospitalization days of ischemic stroke patients and provide positive decision support for patients and doctors. 


\subsection{The importance of predictive characteristics of hospitalization days in patients with Ischemic stroke}

The XGBoost algorithm was used to rank the importance of the features in the prediction of hospitalization days of patients with Ischemic stroke, and the number of times that the features were used as segmentation samples in all cart trees was taken as the importance index. The features including NIHSS, MRS, Hemiplegia aphasia, age, BMI and TIA etc. were found to be the top ten features in importance in predicting the LOS of ischemic stroke patients. The results was shown in Table 5.

The result showed that the four features of admission NIHSS value, admission MRS value, whether hemiplegic aphasia and age of patients had the most significant impact on predicting LOS of ischemic stroke patients, and their essential characteristics were far more significant. Among other features, NIHSS and MRS value on admission were particularly important for the hospitalization days of ischemic stroke patients, ranking first and second, respectively. Simultaneously, it was noted that patients' demographic characteristics, such as age, BMI, marital status, also had a more significant impact. Overall, the aspects of demographic features, past medical history, admission examination characteristics, and operation characteristics had an essential influence on the LOS of patients with ischemic stroke. The features of admission examination and operation greatly influenced the hospitalization time of patients with ischemic stroke, which showed that the length of stay in patients with ischemic stroke was a comprehensive problem mainly affected by the degree of illness of patients was determined by various factors.

\section{Discussion}

This study aimed to develop a new machine learning-based model for predicting LOS at admission time for cardiac patients. The results indicated that the prediction models developed using XGBoost analyses have high value in predicting LOS in Chinese patients with ischemic stroke and the XGBoost Algorithm was an appropriate methane learning method for predicting LOS.

Machine learning methods had been successfully implemented in analyzing the disease risk factors using structured data and had provided unique potential benefits. The results showed that the essential attributes that have the most decisive influence on the LOS were: NIHSS, MRS, Hemiplegia aphasia, age, BMI, TIA, Peripheral arterial disease, operation or not, marital status. Several previous studies had explored the key risk factors for $\operatorname{LOS}[15,23,24]$. The study developed a model for predicting LOS, which provide useful information for physicians or patients to better predict patients' LOS, and help the administrative department to better plan the workflow and work plan of the hospital.

In the cardiac field, discovering data and relationships among various factors enables constructing a model that can predict LOS accurately. A precise estimation of the patients' LOS would be of significant value for systematically managing hospital unit resources (medication, equipment and beds) and personnel distribution [25]. It also plays an important role for performance evaluation purposes which represents important aspects in the new trend of performance-based budgeting. 
Previous studies showed that no model fits all the requirement of predicting unexpected LOS due to the complexities of the various conditions [16, 17, 22]. More attention to outlier cases based on prediction at the preadmission stage results in the reduction of bed transfers during hospitalization. Further, understanding LOS features and comparing them to other patients with similar cases and shorter LOS was essential to address the variation in processes that lead to higher LOS. Having a better understanding of the features enables the development of more effective action plans to eliminate unnecessary procedures that would result in reduced LOS. Accordingly, it would reduce cost, decrease the undue burden on patients and healthcare providers. As a result of providing accurate prediction, hospital and health care system management will be more efficient.

The model was characterized by a small number of attributes (28 attributes) yet provided an accurate assessment of the LOS. Besides, we were able to effectively predict the LOS using prospective data collected at admission time. Thus, the model was simple, accurate, and could be easily adopted in practice. It achieved a high prediction accuracy superior to the results of models adopted in previous studies. Therefore, the model established in this study has a strong practical value, which can well predict the length of stay, and then help hospital administrative staff and doctors to plan their working hours, and help patients and their families to make a good life planning.

However, there were several limitations in the study that need to be recognized. First, the population included in this study covers one prefecture level city in northern China that might negatively impact the performance of the model. In contrast, data from other city or applying the model in other health organizations might have a different result. Second, only two years of patients' records were collected in the study. The external environment changes in recent two years (such as medical insurance payment, public hospital reform system, etc.) might significantly impact on the LOS of patients and, to a certain extent, affect the accuracy of the model.

\section{Conclusion}

The XGBoost algorithm was an appropriate machine learning method for predicting the LOS of patients with ischemic stroke. In the ML model on predicting the LOS of ischemic stroke patients, demographic characteristics, past medical history, admission examination characteristics, and operation characteristics significantly impacted hospitalization days. Based on the prediction model, an intelligent medical management prediction system could be developed to predict the LOS based on electronic medical records of ischemic stroke patients.

\section{Abbreviations}

LOS: length of hospital stay; ML: machine learning; ACC: Accuracy; RE: Recall Rate XGBoost: Extreme Gradient Boosting; TIA: Transient ischemic attack; MRS: Modified Rankin Scale; NIHSS: National Institute of Health stroke scale; 


\section{Declarations'}

\section{Ethics approval and consent to participate}

The study complied with the Helsinki Declaration of 1975, revised in 2008. The study was approved by the School of Social Development and Public Policy of Beijing Normal University Ethics Committee (Protocol Number: SSDPP-HSC 2019004). All analyses were performed on routinely collected anonymized data from the participating institutions.

\section{Consent for publication}

Not applicable.

\section{Availability of data and materials}

Due to privacy laws and the data user agreement among the Cangzhou Central Hospital, Hebei University of Technology and Beijing Normal University, the datasets used and/or analyzed during the current study are available from the corresponding author on reasonable request.

\section{Competing interests}

The authors declare that they have no conflict of interest.

\section{Funding}

The study was funded by the National Key R\&D Program of China (NO: 2018YFB2101100) for Beijing Normal University and the Natural Science Foundation of Hebei Province (NO: G2019202350) for Hebei University of Technology. Both of Beijing Normal University and Hebei University of Technology participated in the research design, data collection, data processing and manuscript writing. Data analysis was mainly completed by Hebei University of technology. Beijing Normal University played a more important role in data interpretation.

\section{Authors' contributions}

$\mathrm{RC}$ and ZSF were responsible for the study design and results; RC and ZSF collected and processed the data; RC, LJ and GDW were responsible for model construction and optimization; ZSF and RC wrote the main manuscript text; ZWJ, TDH, GLN, QZY, and WXH gave suggestions for revising this draft. All the authors contributed to the final manuscript.

\section{Acknowledgements}

Thanks to Refined management office of Cangzhou Central Hospital in Hebei Province, China, who provided us with the electronic medical records. We would also like to thank the clinicians of Cangzhou Central Hospital for their valuable opinions on our data processing.

\section{Authors' details}

${ }^{1}$ School of economics and management, Hebei University of Technology, Tianjin, 
China; ${ }^{2}$ School of Law, Beijing Normal University, Beijing, China; ${ }^{3}$ School of Social Development and Public Policy, Beijing Normal University, Beijing, China;

\section{References}

1. Collaborators GBDCoD: Global, regional, and national age-sex specific mortality for 264 causes of death, 1980-2016: a systematic analysis for the Global Burden of Disease Study 2016. Lancet 2017, 390:1151-1210.

2. Gao JJ, Yu F, Xu ZH, Duan J, Cheng Q, Bai LJ, Zhang YW, Wei QN, Yi WZ, Pan RB, Su H: The association between cold spells and admissions of ischemic stroke in Hefei, China: Modified by gender and age. Science of the Total Environment 2019, 669:140-147.

3. Feigin VL, Forouzanfar MH, Krishnamurthi R, Mensah GA: Global burden of stroke: an underestimate Reply. Lancet 2014, 383:1205-1206.

4. Feigin VL, Nguyen G, Cercy K, Johnson CO, Alam T, Parmar PG, Abajobir AA, Abate KH, Abd-Allah F, Abejie AN, et al: Global, Regional, and Country-Specific Lifetime Risks of Stroke, 1990 and 2016. New England Journal of Medicine 2018, 379:2429-2437.

5. Li X ZX: Annual data set on national mortality surveillance 2018(in Chinese). Beijing: China Science and Technology Press; 2019.

6. Krishnamurthi RV, Feigin VL, Forouzanfar MH, Mensah GA, Connor M, Bennett DA, Moran $\mathrm{AE}$, Sacco RL, Anderson LM, Truelsen T, et al: Global and regional burden of first-ever ischaemic and haemorrhagic stroke during 1990-2010: findings from the Global Burden of Disease Study 2010. Lancet Global Health 2013, 1:259-281.

7. Donnan GA: Navigating 2018, a year in stroke! International Journal of Stroke 2018, 13:5-5.

8. Lekander I, Willers C, von Euler M, Lilja M, Sunnerhagen KS, Pessah-Rasmussen H, Borgstrom F: Relationship between functional disability and costs one and two years post stroke. Plos One 2017, 12.

9. Wang WZ, Jiang B, Sun HX, Ru XJ, Sun DL, Wang LH, Wang LM, Jiang Y, Li YC, Wang YL, et al: Prevalence, Incidence, and Mortality of Stroke in China Results from a Nationwide Population-Based Survey of 480687 Adults. Circulation 2017, 135:759-+.

10. SUN Hai-xin WWz: A nationwide epidemiological sample survey on cerebrovascular disease in China (in Chiese). Chin J Contemp Neurol Neurosurg 2018, 18:6.

11. Group RospatiCW: Brief report on stroke prevention and treatment in China(in Chinese). Chin J Cerebrovasc Dis 2020, 17:10.

12. WANG Xue ZS-y: Hospitalization Expense for Patients with Cerebral Infarction: Based on Degree of Structural Variation and Grey Correlation. (in Chinese) Chin J Rehabil Theory Pract 2020, 26:115-119.

13. Yuan-li CY-nGT-jLJ-1QY-mWBL: Analysis on the hospitalization costs differences and its effects on health outcome among the stroke patients(in Chinese). Chinese Journal of Health Policy 2016, 9:7.

14. CHEN Shuang LP-k, LU Hu- ying, GUO Xiu-hua: Factors Related with Length of Stay for Inpatient Rehabilitation after Stroke(in Chinese). Chin J Rehabil Theory Pract 2014, 20:5.

15. Turgeman L, May JH, Sciulli R: Insights from a machine learning model for predicting the hospital Length of Stay (LOS) at the time of admission. Expert Systems with Applications 2017, 78:376-385. 
16. Carter EM, Potts HWW: Predicting length of stay from an electronic patient record system: a primary total knee replacement example. Bmc Medical Informatics and Decision Making 2014, 14.

17. Tanuja S, Dr. U. Dinesh Acharya, Shailesh K R: Comparison of Different Data Mining Techniques to Predict Hospital Length of Stay. JOURNAL OF PHARMACEUTICAL AND BIOMEDICAL SCIENCES 2011, 7:4.

18. Ke JT, Zheng HY, Yang H, Chen XQ: Short-term forecasting of passenger demand under on-demand ride services: A spatio-temporal deep learning approach. Transportation Research Part C-Emerging Technologies 2017, 85:591-608.

19. Zhang DH, Qian LY, Mao BJ, Huang C, Huang B, Si YL: A Data-Driven Design for Fault Detection of Wind Turbines Using Random Forests and XGboost. Ieee Access 2018, 6:21020-21031.

20. Ma XJ, Sha JL, Wang DH, Yu YB, Yang Q, Niu XQ: Study on a prediction of P2P network loan default based on the machine learning LightGBM and XGboost algorithms according to different high dimensional data cleaning. Electronic Commerce Research and Applications 2018, 31:24-39.

21. Jie Li, Qiaoling Lan, Shihao Ma: Assessment on insurance fraud risk in basic medical insurance in the context of big data(in Chinese). Chinese Journal of Health Policy 2018, 12 : 43-50.

22. Luo L, Li J, Lian S, Zeng X, Sun L, Li C, Huang D, Zhang W: Using machine learning approaches to predict high-cost chronic obstructive pulmonary disease patients in China. Health Informatics Journal 2020, 26:1577-1598.

23. Sato K, Arai N, Takeuchi S: Status epilepticus severity score as a predictor for the length of stay at hospital for acute-phase treatment in convulsive status epilepticus. Journal of Clinical Neuroscience 2020, 75:128-133.

24. Huang YC: The impact factors on the cost and length of stay among acute ischemic stroke. Journal of Stroke \& Cerebrovascular Diseases 2013, 7:152-158.

25. Badaki-Makun O, Casella JF, Tackett S, Tao XT, Chamberlain JM: Association of Antibiotic Choice With Hospital Length of Stay and Risk Factors for Readmission in Patients With Sickle Cell Disease and Acute Chest Syndrome: An Observational Cohort Study. Annals of Emergency Medicine 2020, 76:S37-S45.

Table. 1 Variable of the data

Table.2 Main parameters of the model for predicting LOS of ischemic stroke patients

Table. 3 Baseline features

Table. 4 The performance of the model evaluated using 10-fold cross-validation method Table.5 The importance of features of prediction model

Figure. 1 The steps of building the machine learning model to predict the LOS of ischemic stroke patients 
Figures

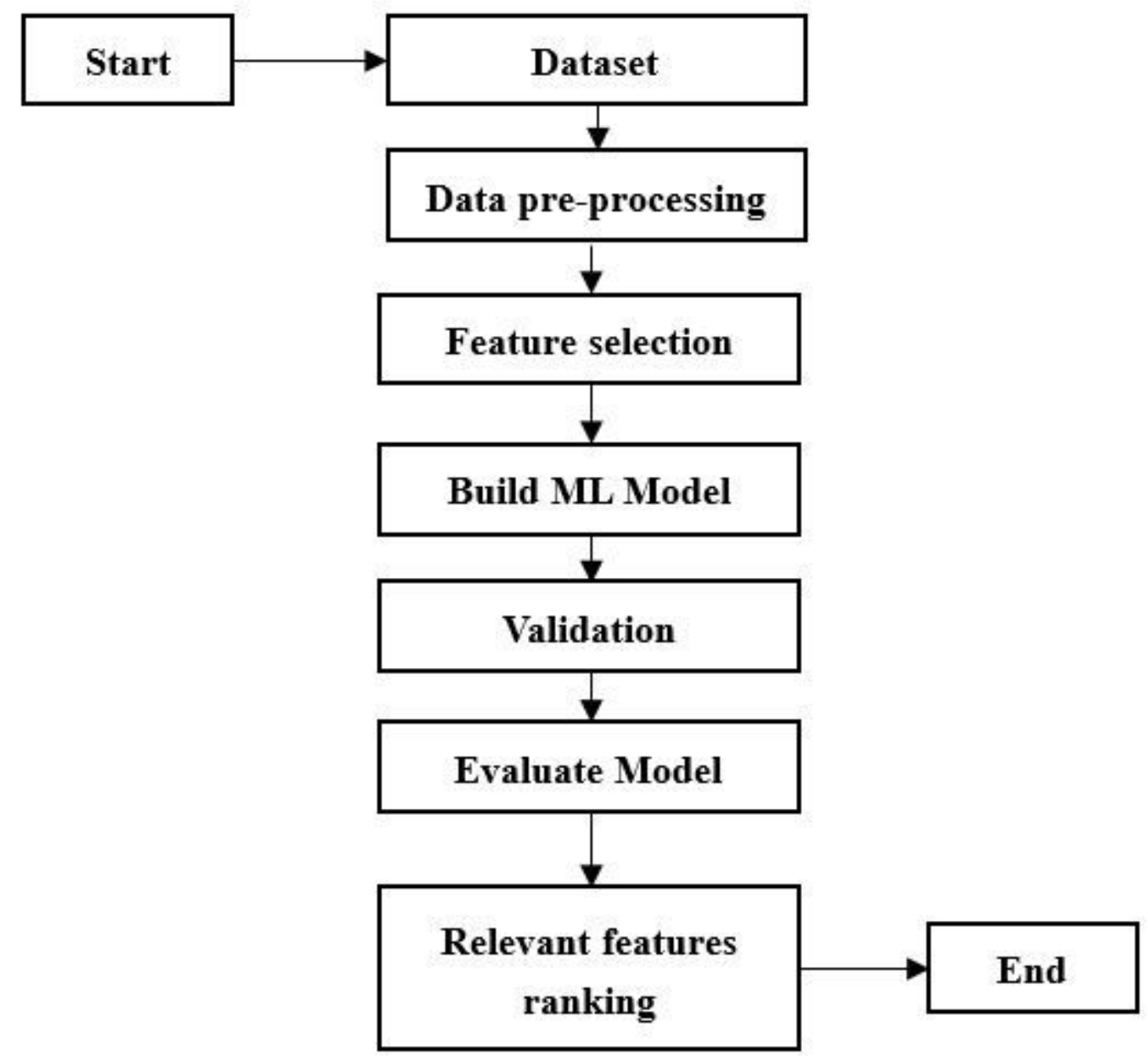

Figure 1

The steps of building the machine learning model to predict the LOS of ischemic stroke patients 